1 Title: Structure of the Human Secretory Immunoglobulin M Core

3 Authors: Nikit Kumar ${ }^{1}$, Christopher P. Arthur ${ }^{1}$, Claudio Ciferri ${ }^{1}$, and Marissa L.

4 Matsumoto $^{1 *}$

6 Affiliations: ${ }^{1}$ Department of Structural Biology, Genentech, 1 DNA Way, South San

7 Francisco, CA 94080, USA. *To whom correspondence should be addressed:

8 matsumoto.marissa@gene.com

\title{
Abstract
}

11 Immunoglobulins (Ig) $\mathrm{A}$ and $\mathrm{M}$ are the only human antibodies that form oligomers and

12 undergo transcytosis to mucosal secretions via the polymeric Ig receptor (plgR). When

13 complexed with the J-chain (JC) and the secretory component (SC) of plgR, secretory

$14 \lg \mathrm{A}$ and $\lg \mathrm{M}$ (slgA and slgM) play critical roles in host-pathogen defense. Recently, we

15 determined the structure of $\operatorname{sigA}-F_{c}$ which elucidated the mechanism of polymeric $\lg \mathrm{A}$

16 assembly and revealed an extensive binding interface between IgA-Fc, JC, and SC.

17 Despite low sequence identity shared with IgA-Fc, IgM-Fc also undergoes JC-mediated

18 assembly and binds plgR. Here, we report the structure of slgM-Fc and carryout a

19 systematic comparison to slgA-Fc. Our structural analysis reveals a remarkably

20 conserved mechanism of JC-templated oligomerization and SC recognition of both IgM

21 and $\lg A$ through highly a conserved network of interactions. These studies reveal the 
22 structurally conserved features of slgM and slgA required for function in mucosal

23 immunity.

25 Introduction

The human immune system is composed of five major classes of

27 immunoglobulins (lg). IgG, $\lg \mathrm{D}$, and $\lg \mathrm{E}$ exist only as monomers, whereas $\lg A$ and $\lg \mathrm{M}$

28 have the ability to form polymers due to an 18 amino acid tailpiece extension on their

29 heavy chains (Sørensen et al., 1996). IgA dimers, tetramers, and pentamers can form

30 only in the presence of the J-chain (JC), a $15 \mathrm{kDa}$, cysteine-rich polypeptide required for

31 its polymeric assembly (Johansen et al., 2000). In contrast, polymeric IgM can form both

32 pentamers and hexamers, depending on the presence or absence of the $\mathrm{JC}$,

33 respectively (Heyman and Shulman, 2016; Kownatzki and Drescher, 1973).

Hexameric IgM is best known for its role in complement activation through the

35 classical pathway via C1q (Sharp et al., 2019), whereas pentameric IgM plays an

36 important role in host pathogen defense at the mucosa (Johansen et al., 1999). Like

37 polymeric $\lg \mathrm{A}, \mathrm{JC}$-containing, pentameric $\operatorname{lgM}$ binds to the polymeric $\lg$ receptor (plgR)

38 and can be transcytosed across the epithelium to carry out its protective function in

39 mucosal tissues (Johansen et al., 2000). Upon transcytosis the plgR is proteolytically

40 cleaved releasing the ectodomain of the receptor known as the secretory component

41 (SC), which remains associated with IgA and IgM (Johansen et al., 2000). The released

$42 \lg$-SC complex is referred to as secretory $\lg A$ or $\lg M(\operatorname{sg} A$ or slgM). While slgA is the

43 predominant immunoglobulin found in mucosal secretions, slgM plays an important role, 
44 particularly highlighted by the fact that selective IgA deficiency does not typically result

45 in recurrent infections due to the ability of slgM to provide protection (Catanzaro et al.,

46 2019).

Despite extensive study, the structures of slgA and slgM have remained elusive

48 for many years. Recently we determined the first atomic-resolution structures of dimeric,

49 tetrameric, and pentameric assemblies of slgA-Fc (Kumar et al., 2020). Our structures

50 revealed the novel fold of the $\mathrm{JC}$, which acts as a template for $\operatorname{lgA}-\mathrm{Fc}$ oligomerization

51 mediated by the tailpieces. The interaction of the JC with the IgA-Fcs imparts

52 asymmetry on the IgA oligomer allowing one-to-one binding with the SC. The SC binds

53 across the IgA oligomer making extensive interactions with both the JC and Fcs.

54 Furthermore, the structures revealed that the SC undergoes a large conformational

55 rearrangement from a closed, apo state (Stadtmueller et al., 2016), to an open,

56 extended conformation upon polymeric IgA binding. Our structures are highly consistent

57 with two additional reported structures of murine

58 (https://doi.org/10.1101/2020.02.16.951780) and human slgA (Wang et al., 2020).

59 To better understand whether the mechanism of polymerization and SC

60 recognition is conserved across both secreted lg isotypes, we determined the structure

61 of the human slgM-Fc core by cryo-electron microscopy (cryo-EM) and carried out a

62 detailed comparative analysis with the structure of slgA-Fc. Although slight differences

63 in the flexibility of the JC are observed along with minor differences in binding by the

64 SC, the architecture of slgA and slgM is highly homologous and consistent with the

65 recently reported structure of slgM by Li and colleagues (Li et al., 2020). Our structures 
reveal a mechanism of JC-mediated oligomerization that is remarkably conserved in

67 both slgA and slgM. Preservation of the molecular interactions required for SC

68 recognition between $\lg \mathrm{A}$ and $\lg \mathrm{M}$ suggests highly similar plgR binding and transcytosis

69 mechanisms for both secreted Igs. These studies reveal the highly conserved nature of

70 secreted polymeric Ig assembly, structure, and function.

71

72

73 Results

74

Cryo-EM Structure of the Secretory Immunoglobulin M Core

Prior analysis of recombinant full-length pentameric IgM by negative stain EM

77 revealed significant flexibility of the Fab arms (Hiramoto et al., 2018). In order to obtain

78 a rigid assembly for structural determination, full-length IgM was truncated to $\lg M-F c$

79 containing only the heavy chain constant domains $(\mathrm{C} \mu 2, \mathrm{C} \mu 3$, and $\mathrm{C} \mu 4)$ and the

80 tailpiece. IgM-FC was co-expressed with the JC to obtain a pentameric assembly. To

81 further rigidify the pentamers, a complex was formed with the secretory component (SC)

82 of the plgR and single particle cryo-EM was used to obtain a 3D reconstruction (Fig. 1 -

83 figure supplement 1 and Table 1). The sample displayed significant preferential

84 orientation, which was overcome by collecting additional data at a tilt angle of 40

85 degrees. Data from zero and 40 degree tilt were processed to yield a $3.25 \AA$

86 reconstruction [Fourier shell correlation $(\mathrm{FSC})=0.143$ ] of the complex that included the

$87 \mathrm{C} \mu 3, \mathrm{C} \mu 4$, and tailpiece segments of the IgM-Fc, the JC, and the SC. Despite C $\mu 2$ 
88 being included in the construct, flexibility of this domain precluded structure

89 determination. Regions of the map containing the highest local resolution were at the

90 complex core and included the tailpiece segments as well as the interface between the

91 SC domain 1 (D1), IgM C $\mu 4$, and the JC (Fig. 1 - figure supplements 2 and 3).

92 Homology models of the human $\lg \mathrm{M} \mathrm{C \mu 3}, \mathrm{C} \mu 4$, and tailpieces were built based on the

93 NMR structure of murine $\mathrm{C} \mu 3$ (PDB code 4BA8), the crystal structure of murine $\mathrm{C} \mu 4$

94 (PDB code 4JVW), and the tailpiece segments from pentameric slgA-Fc (PDB code

95 6UEA), respectively. These models along with the JC and individual domains of the SC

96 from pentameric slgA-Fc (PDB code 6UEA) were docked, rigid-body fit into the map,

97 and refined.

98 Overall, slgM forms an asymmetric pentamer, with the J-chain occupying the site

99 where a sixth IgM-Fc would be expected in a symmetric, hexameric assembly (Fig. 1).

100 This observation is in line with previously reported negative stain analysis of IgM-Fc

101 complexed with JC and the apoptosis inhibitor of macrophage molecule (AIM) (Hiramoto

102 et al., 2018). The five Fcs lie in-plane, stabilized by inter-Fc disulfide bonds between

103 C414 in the CH3 domain of adjacent Fcs, as suggested previously (Davis et al., 1989;

104 Sørensen et al., 1999). Additionally, the Fcs are arranged radially around a central $\beta$ -

105 sandwich composed of the JC and the tailpieces of the five Fcs. The SC binds across

106 the top face of the pentamer in a bent, but extended conformation, contacting Fc1, Fc2

107 and the JC. The overall architecture of slgM-Fc is strikingly reminiscent of pentameric

108 slgA-Fc ((Kumar et al., 2020), PDB code 6UEA). 
A
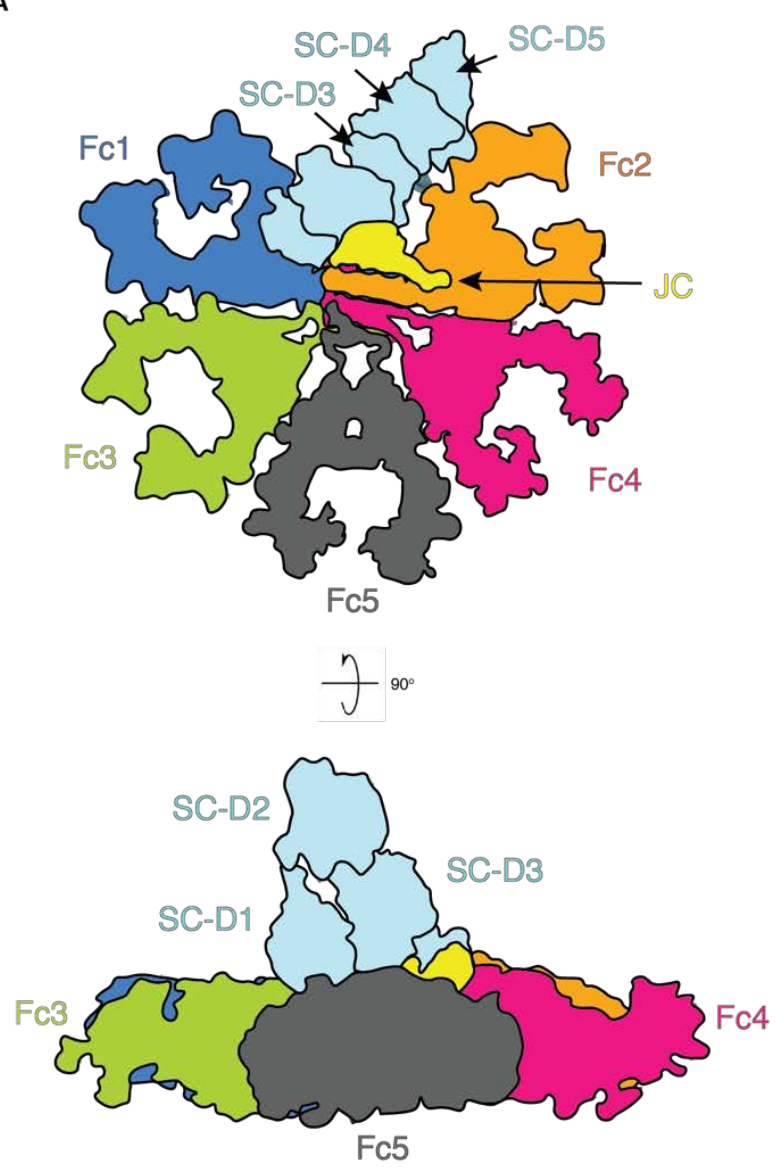

$$
\checkmark 90^{\circ}
$$

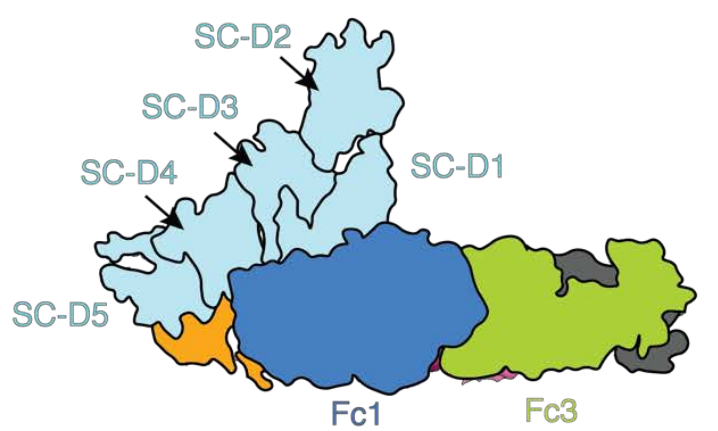

B

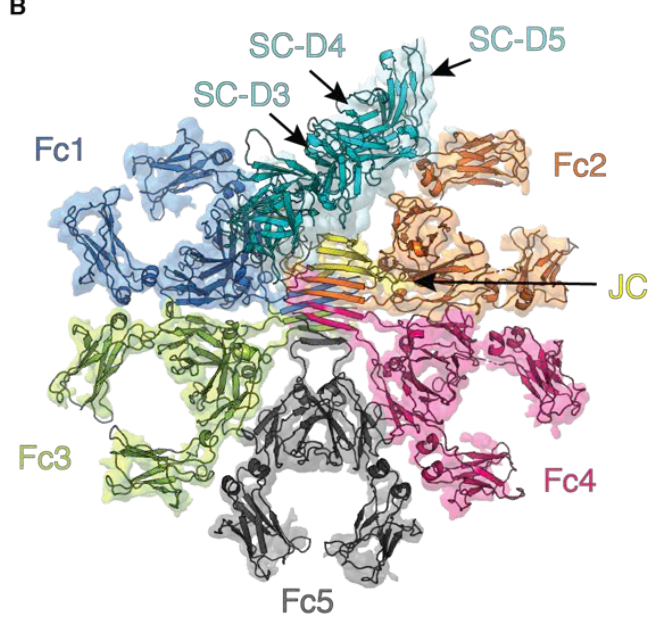

$\eta_{90^{\circ}}$

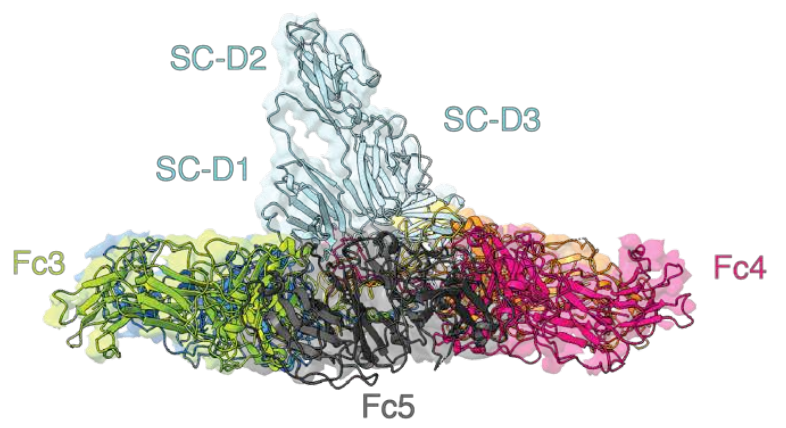

$\mho_{90^{\circ}}$

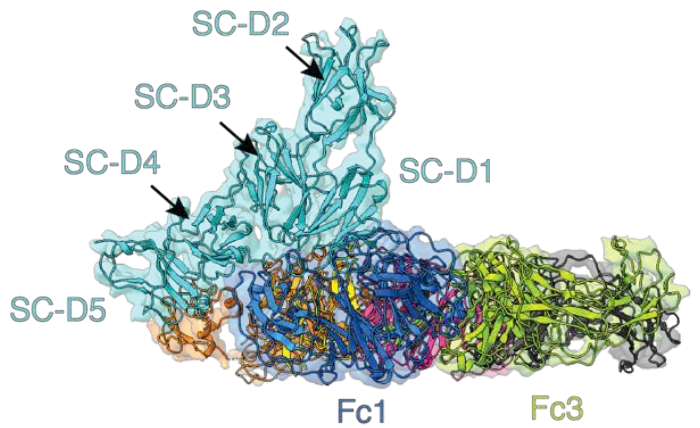

Fc1
FC3

Figure 1: Cryo-EM structure of the pentameric slgM complex. (A) Top, front, and side schematics of the slgM complex. (B) Transparent cryo-EM maps overlaid with the model are shown for the complex as in (A). 


\section{Structure of the J-chain}

With an overall resolution of $3.25 \AA$ and the highest local resolution at the core of

113 the IgM-Fc/JC/SC interaction, the JC model from our previous slgA structure could be

114 easily docked into the map. A major difference between the slgA and slgM structures is

115 that in slgM, we did not observe density for residues 71-98 of the 137-residue JC (Fig.

116 2A). This is consistent with the structure reported by Li and colleagues (Li et al., 2020).

117 In IgA, as previously characterized, the JC interacts with the Ca3 domains of $\lg A$

118 through its three $\beta$-hairpin regions, with $\beta$-hairpins 1 and 2 interacting on the top surface

119 of Fc2, while $\beta$-hairpin 3 interacts with the bottom surface of Fc1 (Fig. 2B). While $\beta$ -

120 hairpins 1 and 3 are similarly observed to interact with the $C \mu 4$ domains of IgM to the

121 top and bottom surfaces of Fc2 and Fc1, respectively, residues 71-98 corresponding to

$122 \beta$-hairpin 2 were unstructured (Fig. 2A). JC $\beta$-hairpin 1 interacts with IgM via the

123 packing of $\mathrm{JC}_{122}$ and $\mathrm{JC}_{\mathrm{V} 34}$ with $\operatorname{lgM} \mathrm{Fc} 2$ and anchored by a salt-bridge interaction

124 between $\mathrm{JC}_{\mathrm{E} 31}$ with IgM FC2 $\mathrm{R}_{461}$ (Fig. 2C). In pentameric $\lg \mathrm{A}, \mathrm{JC}_{\mathrm{I} 22}$ and $\mathrm{JC}_{\mathrm{V} 34}$ similarly

125 pack against IgA Fc2, while the tip of the $\beta$-hairpin is anchored by a salt-bridge

126 interaction between $\mathrm{JC}_{\mathrm{D} 32}$ and $\lg \mathrm{A} \mathrm{FC}_{\mathrm{R} 450}$ (Fig. 2C). Interestingly, while IgMR461 is not

127 conserved in IgA (Fig. 2 - figure supplement 1A), the salt-bridge interaction is

128 maintained by a neighboring residue, $\lg \mathrm{A}_{\mathrm{R} 450}$, located near this interface. Also, instead

129 of forming the salt-bridge with $\mathrm{JC}_{\mathrm{E} 31}$, IgA instead uses the adjacent residue $\mathrm{JC}_{\mathrm{D} 32}$. JC $\beta$ -

130 hairpin 3 primarily packs residues JCV114, L116, Y118, V125 against hydrophobic IgM

131 residues Fc1 L359, F485, V537, v547 in a groove formed between $\mathrm{C} \mu 3$ and $\mathrm{C} \mu 4$ of IgM Fc1

132 (Fig. 2D). This interaction is similar to the interaction of JC $\beta$-hairpin 3 with $\lg A$ where 
bioRxiv preprint doi: https://doi.org/10.1101/2020.09 10.291138; this version posted September 11,2020 . The copyright holder for this preprint (which was not certified by peer review) is the author/funder, who has granted bioRxiv a license to display the preprint in perpetuity. It is made available under aCC-BY 4.0 International license.

A

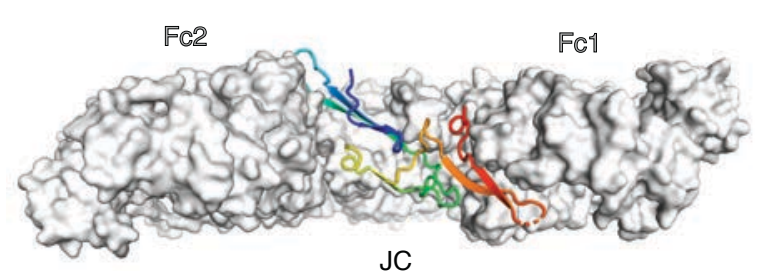

$\mathrm{JC}$

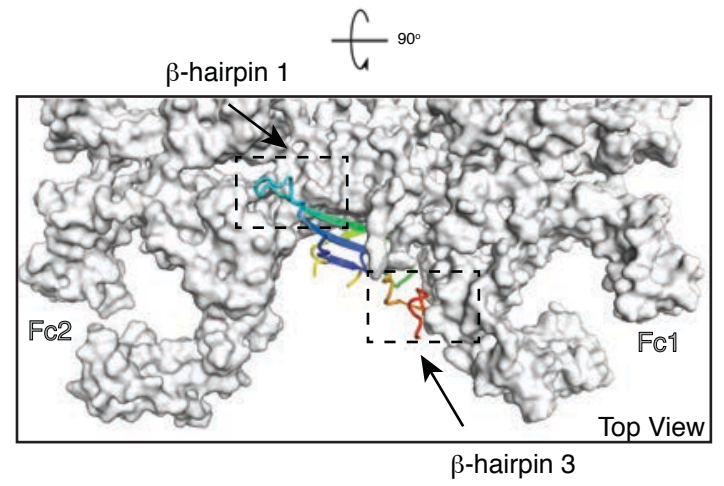

B
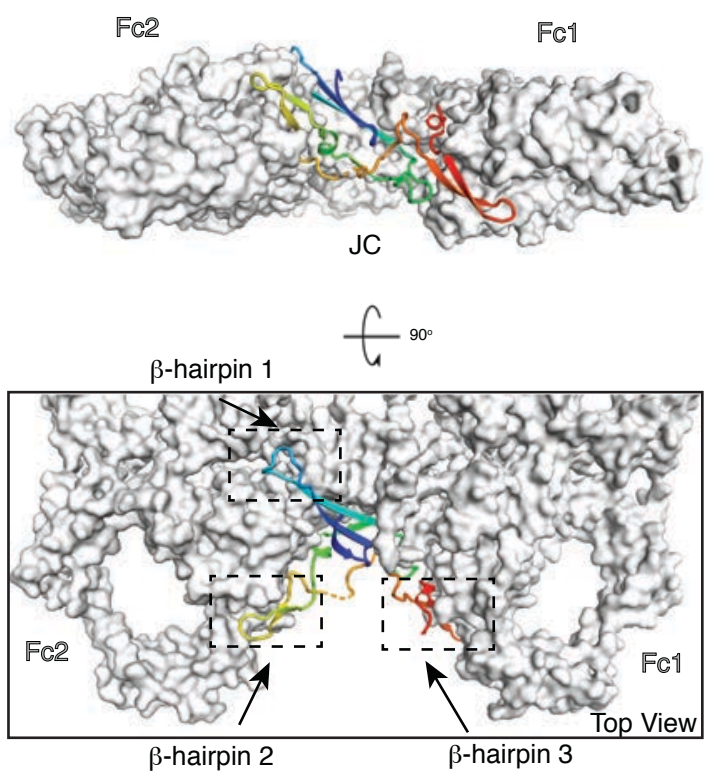

E

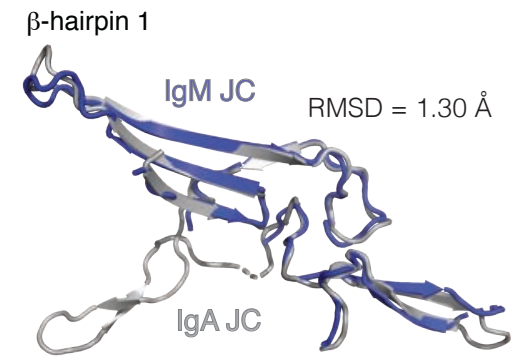

$\beta$-hairpin 2

$\lg \mathrm{A}$

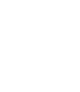

D

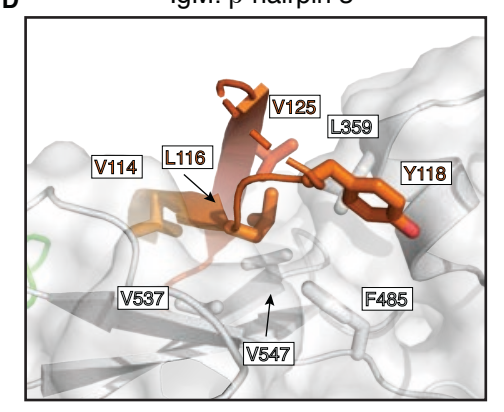

gM: $\beta$-hairpin 3 $\lg A$ : $\beta$-hairpin 1
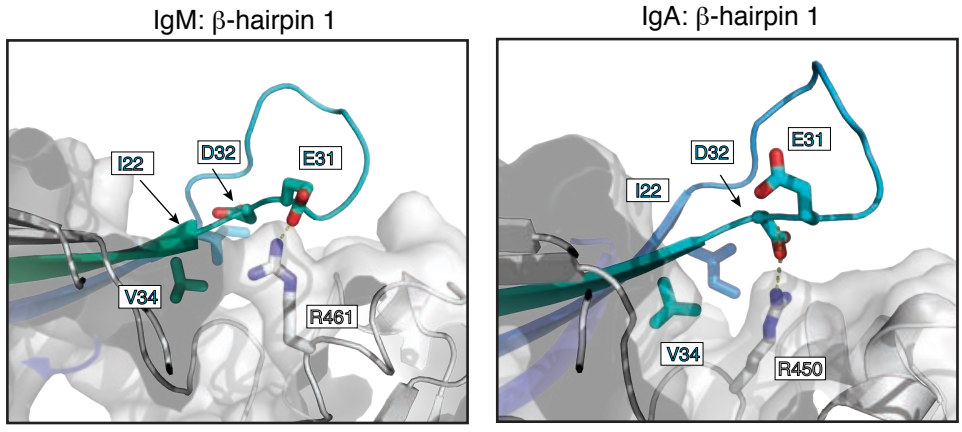

$\lg \mathrm{A}: \beta$-hairpin 3

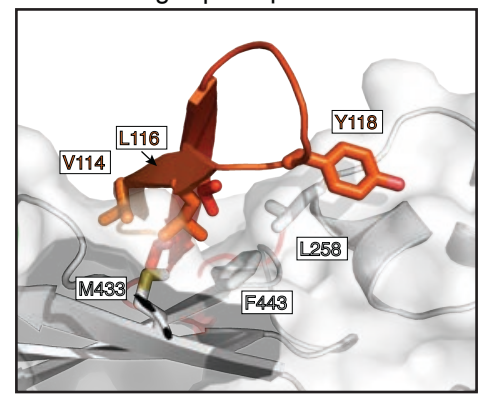


$134 \mathrm{JCV}_{\mathrm{V} 114,}$ L116, Y118, V125 pack against homologous IgA residues FC1 L258, M433, F443 in a

135 hydrophobic groove between domains Ca2 and Ca3 of IgA Fc1 (Fig. 2D). With the

136 exception of $\beta$-hairpin 2, which is unstructured in $\operatorname{sigM}$, the structure of the JC is very

137 similar between pentameric slgM and slgA complexes, with an overall RMSD of $1.30 \AA$ 138 (Fig. 2E).

\section{Mechanism of IgM Polymerization}

In addition to clasping Fc1 and Fc2 of IgM, the JC also templates a central $\beta$ -

142 sandwich structure that is extended by the tailpieces of each IgM Fc. Tailpiece residues

143 L561 to S569 from Fc1 and Fc3 contribute a pair of parallel $\beta$-strands each that extend

144 the bottom face of the central $\beta$-sandwich, while Fc2 and Fc4 contribute a pair of

145 parallel $\beta$-strands each that extend the top face (Fig. 3A). Meanwhile, Fc5 contributes

146 one $\beta$-strand to both the top and bottom of the central $\beta$-sandwich and effectively caps

147 this assembly, resulting in a $\beta$-sandwich structure containing parallel $\beta$-strands on each

148 surface with an anti-parallel arrangement of the top and bottom sheets. This assembly

149 is identical to the central $\beta$-sandwich assembly of the IgA pentamer (Fig. 3B). Despite

150 minor differences in individual amino acid composition of the tailpieces (Fig. 2 - figure

151 supplement $1 \mathrm{~A}$ ), the arrangement results in a striking array of repeated residues across

152 the $\beta$-sandwich, with primarily hydrophobic residues packed in the center (including IgM

153 FCY562, V564, L566, M568 as compared to IgA FCI458, V460, V462, M464), while hydrophilic residues

154 remain solvent exposed (including IgM F $\mathrm{CN}_{\mathrm{N} 563, \mathrm{~S}} 565$ as compared to $\lg \mathrm{A} \mathrm{FC}_{\mathrm{H} 457, \mathrm{~N} 459,}$, 5461)

155 (Fig. 3C-D). 
A

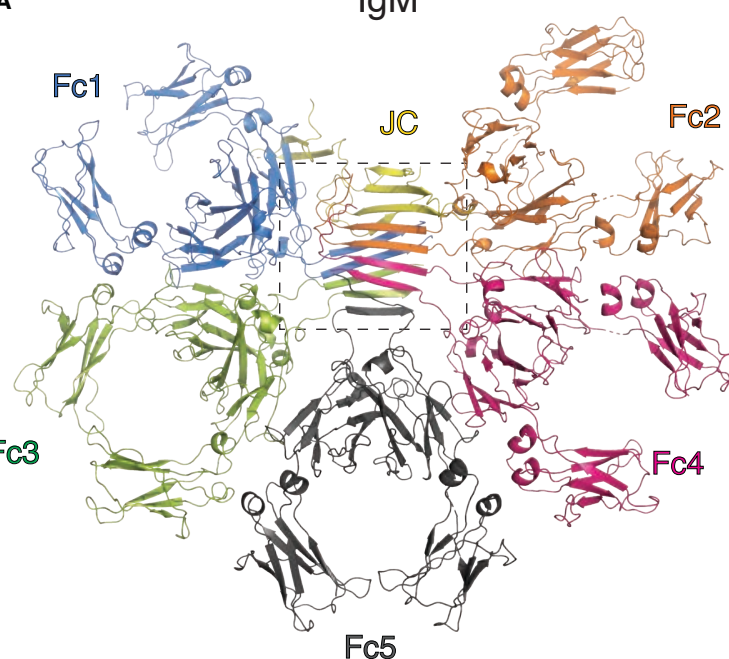

C

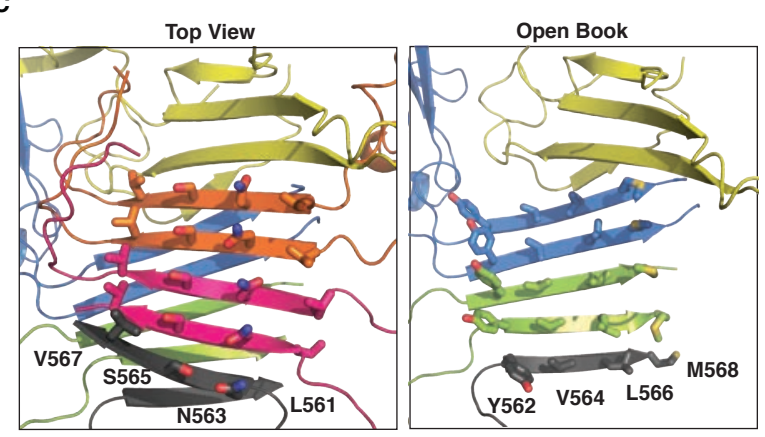

B

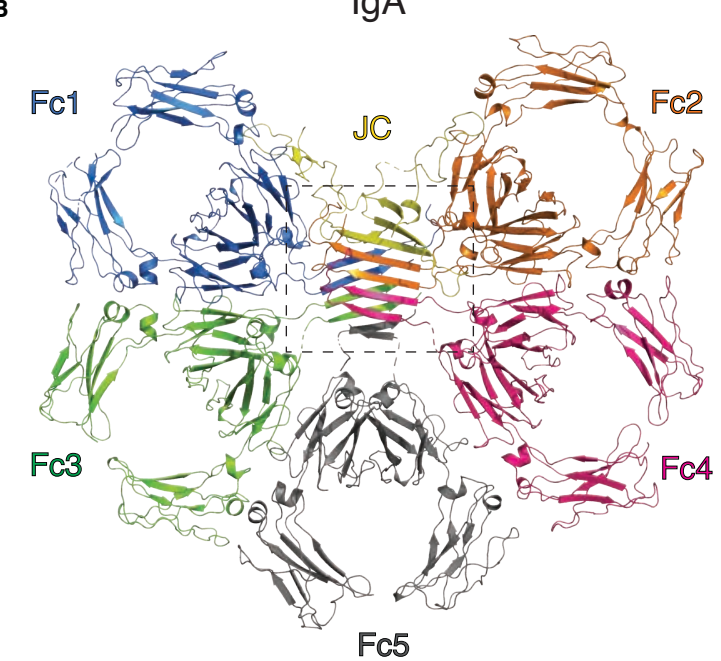

D

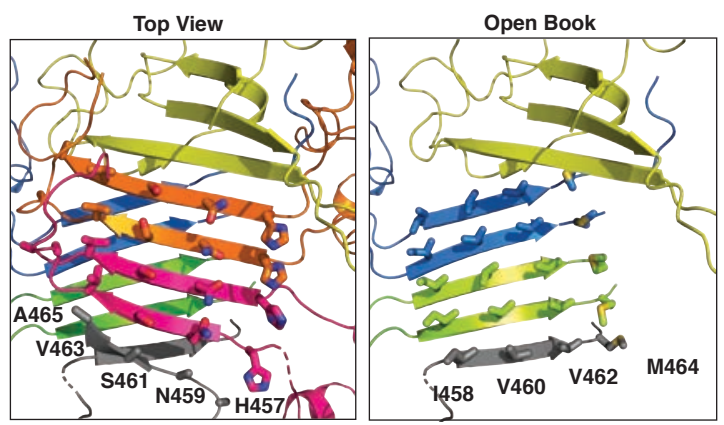

Figure 3: Comparison of tailpiece segments in the oligomerization of pentameric $\lg M$ and $\lg A$. Top views of (A) pentameric IgM and (B) pentameric IgA represented without the SC. (C) Magnification of IgM boxed region in (A) with solvent facing, repeating residues on the top surface of the tailpiece segments of the Fcs (left) and open book view with inward facing, repeating residues labelled and shown as sticks. (D) Similar representation as in (C) for IgA with repeating residues labelled and shown as sticks on top surface (left) and inner surface (right). (B,D) Modified from Kumar et al., 2020 with permission from AAAS.

\section{Recognition of IgM by the Secretory Component}

The transport of IgM across epithelial cells requires the recognition by the plgR.

The SC of the plgR is composed of five Ig-like domains (D1 - D5) (Stadtmueller et al., 2016), and previous biochemical investigations established the involvement of the three CDR-like loops of D1 as necessary for interaction with JC-containing, polymeric IgM 
164 completely buried in an interface between the D1-D4-D5 domains (Stadtmueller et al.,

165 2016). When bound to pentameric IgM, SC D2-D5 are placed head-to-tail in a linear

166 arrangement, while the linker between D1-D2 is turned nearly $180^{\circ}$ to position D1 for

167 interaction with IgM Fc1 (Fig. 4A). SC-D1 is responsible for the bulk of the interactions

168 with the IgM Fcs and the JC through its three CDR-like loops (Fig. 4B), while SC-D5

169 packs against IgM Fc2. Interestingly, while a disulfide bond is clearly formed between

$170 \mathrm{SC}_{\mathrm{C} 468}$ and $\operatorname{lgA} \mathrm{FC}_{\mathrm{C} 311}$ in slgA, weak density between $\mathrm{SC}_{\mathrm{C} 468}$ and the homologous

171 residue in IgM, Fc2c414 was observed at high contour levels of the map, suggesting

172 partial oxidation of the disulfide in our slgM structure. A covalent interaction between the

$173 \mathrm{SC}$ and IgM/JC assemblies has been previously reported (Longet et al., 2012).

174 D1 of the SC recognizes pentameric IgM and IgA through a network of

175 conserved interactions involving all three of its CDR-like loops. In slgM, CDR1 interacts

176 via a salt-bridge between $\mathrm{D}_{\mathrm{R} 31}$ and $\mathrm{JC}_{\mathrm{D} 137}$ along with cation-pi stacking interactions

177 between $\mathrm{D} 1 \mathrm{H} 32_{2}$ with $\mathrm{JC}_{\mathrm{Y} 135}$, which in turn packs favorably against IgM FC1P544.

178 Additionally, $\mathrm{JC}_{\mathrm{D} 132}$ forms a salt-bridge with IgM FC1R451 (Fig. 4C). The mechanism of

179 CDR1 recognition is conserved in slgA with identical JC interactions (with a slightly

180 different rotamer conformation for D1R31) and packing against the homologous $\lg A$

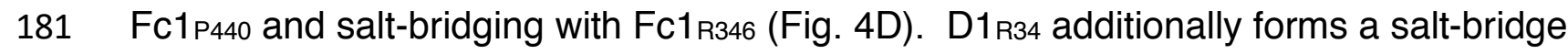

182 with IgM Fc1E468 (Fig. 4E), which is conserved in slgA with Fc1E363 (Fig. 4F). CDR2

183 makes backbone interactions between D1E53 and IgM Fc1R514, along with favorable

184 packing of D1Y55 against Fc1s469 (Fig. 4E). While these exact CDR2 interactions are not

185 conserved in slgA, similar compensatory interactions are formed. Backbone interactions 
A

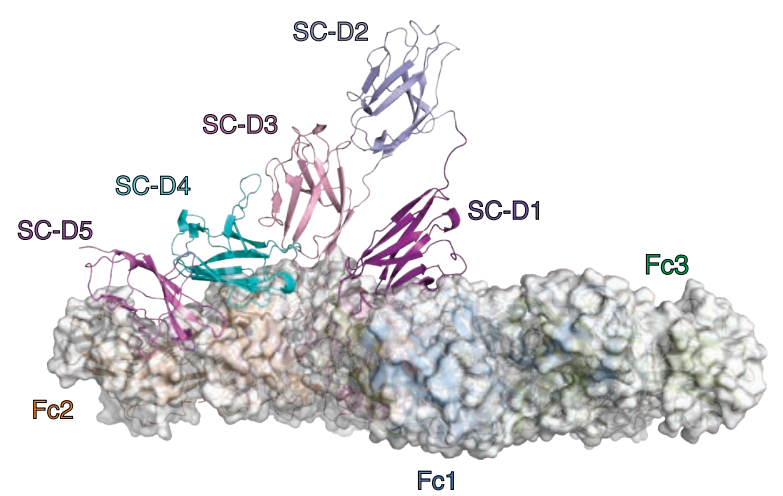

B

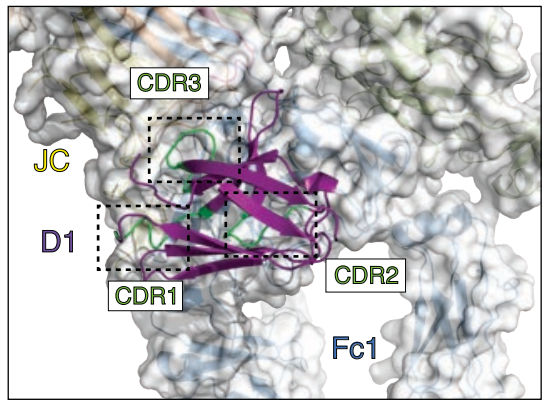

C
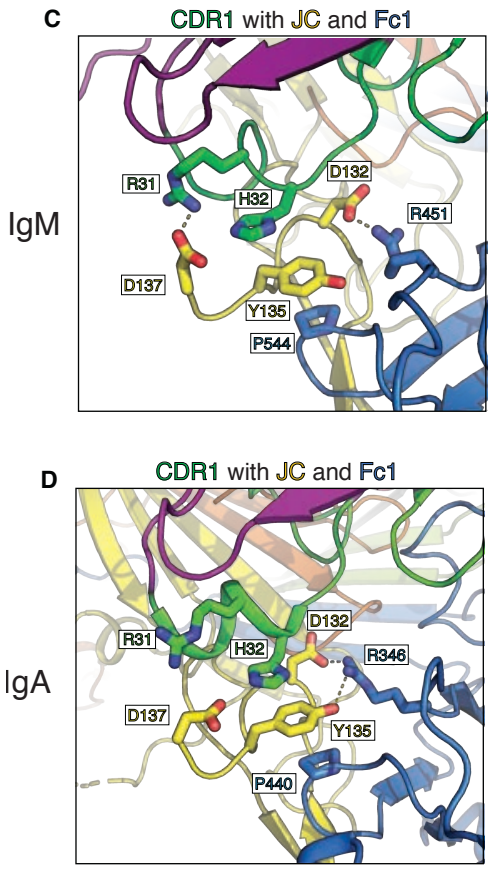

E

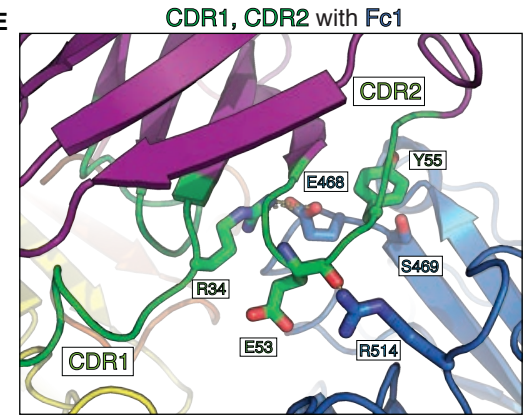

$\mathbf{F}$

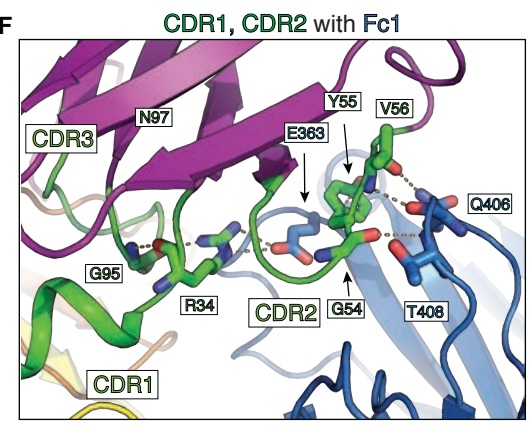

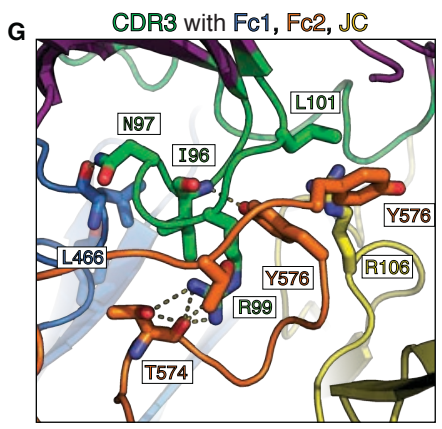

H

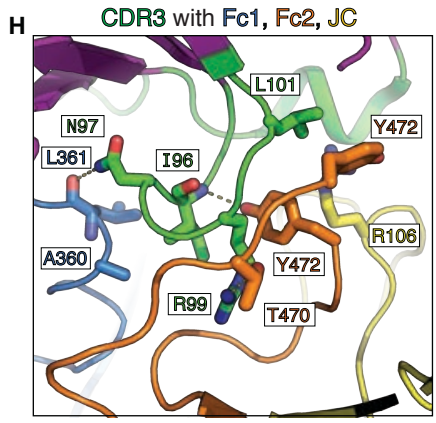

Figure 4: Recognition of pentameric IgM and IgA by SC. (A) slgM pentamer shown as colored model overlaid with white surface representation of FCs and JC. Individual domains D1 - D5 of the SC are colored differentially. (B) Top-down view of SC-D1 and its interactions with IgM-Fc/JC through its three CDR regions (boxed and colored green). D1-CDR1 interactions with JC and Fc1 for $\lg M(C)$ and $\lg A(D)$. Interactions of D1-CDR1 and -CDR2 with Fc1 of $\lg M(E)$ and $\lg A(F)$. Interactions of D1-CDR3 with Fc1, $\mathrm{Fc}$, and $\mathrm{JC}$ of $\operatorname{lgM}(\mathrm{G})$ and $\lg \mathrm{A}(\mathrm{H})$. Side chains are shown as sticks and polar interactions shown as dashes. (D,F,H) Modified from Kumar et al., 2020 with permission from AAAS. 
190 involving residues from Fc1, Fc2, and the JC in both IgM and IgA complexes.

191 Specifically, the backbone amine of SC196 forms a hydrogen-bond with IgM Fc2Y576,

$192 \mathrm{SC}_{\mathrm{N} 97}$ hydrogen-bonds with the backbone carbonyl of IgM Fc1 L466, while SC $\mathrm{R}_{99}$ interacts

193 with IgM FC2T574 and sandwiches Fc2Y576 with $\mathrm{JC}_{\mathrm{R} 106}$ (Fig. 4G). This mechanism of

194 interaction with $\operatorname{lgA}$ is highly conserved, with analogous interactions between $\mathrm{SC}_{196}$ with

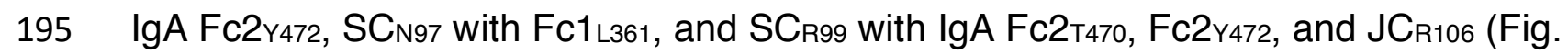

$1964 \mathrm{H})$. Overall, the SC-D1 interaction interface in slgM and slgA is highly conserved,

197 despite sharing an overall sequence identity of only $40 \%$ between the two Fcs (Fig. 2 -

198 figure supplement 1A).

201 Discussion

202

Secreted polymeric Igs play a critical role in host-pathogen defense processes at

203 mucosal surfaces. In this study, we report the structure of the human slgM-Fc core,

204 comprising IgM Fc domains $\mathrm{C} \mu 3$ and $\mathrm{C} \mu 4, \mathrm{JC}$, and the SC, and assess the structural

205 similarities with pentameric slgA. Despite sharing just $40 \%$ sequence identity, both

206 immunoglobulins are able to incorporate the JC and transcytose to mucosal surfaces by

207 interacting with the plgR. A detailed structural comparison between pentameric slgM

208 and slgA reveals that conserved key interaction interfaces with the JC and the SC

209 allows for a remarkably similar assembly of these secreted Igs. These include the

210 association of JC $\beta$-hairpins-1 and -3 with the Ca2-Ca3 and $\mathrm{C} \mu 3-\mathrm{C} \mu 4$ domains, the Fc

211 tailpiece-mediated extension of a central $\beta$-sandwich, and a conserved interface for the 
212 interaction with SC-D1. During the preparation of this manuscript, a $3.4 \AA$-resolution

213 cryo-EM structure of slgM-Fc was also published (Li et al., 2020). Our independent

214 study reveals a highly consistent structure, further validating these novel findings.

While the structures of slgA and slgM are highly similar, we were unable to

216 observe residues corresponding to JC $\beta$-hairpin 2 in slgM, which is also consistent with

217 the observations of $\mathrm{Li}$ and colleagues ( $\mathrm{Li}$ et al., 2020). This was unexpected given the

218 interaction interface between JC $\beta$-hairpin 2 and $\lg$ A-Fc primarily consists of van der

219 Waals interactions and the hydrophobic nature of the structurally homologous residues

220 are generally conserved in IgM-Fc. Perhaps minor charge differences caused by the

221 replacement of $\lg A_{L 441}$ to $\lg M_{N 545}$ at the base of the $\beta$-hairpin 2-binding site or

222 insufficient shape complementarity at the IgM-Fc $C \mu 3-C \mu 4$ interface are responsible.

223 Despite this difference, the remaining JC structure is very similar to that adopted in

224 pentameric slgA with an RMSD of $1.30 \AA$.

225 The conformation of the SC when bound to IgM-Fc or IgA-Fc is nearly identical,

226 with the majority of interactions mediated by the CDR regions of SC-D1, as predicted

227 from mutagenesis studies (Kaetzel, 2005). Interestingly, while we had previously

228 observed clear density for a single disulfide bond linking SC-D5c468 to IgA-Fcc311, we

229 observed weak density for a disulfide between D5c468 and IgM-Fcc414 despite using an

230 identical protocol for complex formation containing reduced glutathione. Previous

231 reports have indicated that the SC is covalently attached to IgM (Longet et al., 2012),

232 while other reports indicate that SC non-covalently interacts with IgM (Hamburger et al.,

233 2004; Stadtmueller et al., 2016). Based on our observations where SC-D5 orients in a 
234 similar conformation when complexed with either $\lg \mathrm{A}-\mathrm{Fc}$ or $\lg \mathrm{M}-\mathrm{Fc}$, along with the close

235 spatial proximity of the respective cysteines, it is likely that there was a mixture of

236 particles in our slgM-Fc sample that either contain the disulfide or those that are non-

237 covalently interacting.

238 Overall, our study provides structural insights into the pentameric assembly of

$239 \operatorname{IgM}-F c$ complexed with the JC and bound to the SC. Both polymeric $\lg M$ and $\lg A$

240 complexed with JC also interact with Fca/ $\mu \mathrm{R}$ with likely overlapping binding sites as SC-

241 D1 (Hamburger et al., 2004), while only lgM interacts with the FchR/Faim3/Toso (Liu et

242 al., 2019). Further structural characterization of the interaction with these receptors

243 would allow for a better understanding of the mechanism of receptor specificity for $\lg M$

244 and provide further insights into the role of $\operatorname{lgM}$ beyond mucosal immunity.

247 Materials and Methods

249 Construct Generation

250 The protein sequence for human IgM-Fc was obtained from UniProt (www.uniprot.org).

251 A sequence encoding residues 226-576 was synthesized and cloned into a mammalian

252 pRK expression vector containing an N-terminal signal sequence, FLAG-tag, and

253 PreScission protease cleavage site. Constructs encoding human JC (residues 23-159)

254 and human SC (residues 19-603 containing a C-terminal hexahistidine tag) was

255 described previously (Kumar et al., 2020; Lombana et al., 2019). 
257 Protein Expression and Purification

258 Vectors encoding IgM-Fc and JC were transiently co-transfected into CHO DP12 cells

259 as previously described (Wong et al., 2010) in a 4:1 DNA mass ratio. Media containing

260 secreted IgM-Fc/JC assemblies was purified by anti-FLAG affinity resin (Genentech,

261 South San Francisco, CA), washed with buffer A (50 mM Tris, pH 7.5, $150 \mathrm{mM} \mathrm{NaCl,} 5$

$262 \mathrm{mM}$ EDTA, and $2 \mathrm{mM} \mathrm{NaN}_{3}$ ), eluted with $50 \mathrm{mM}$ sodium citrate, $\mathrm{pH} 3.5,150 \mathrm{mM} \mathrm{NaCl}$,

263 and neutralized with $0.2 \mathrm{M}$ arginine, $137 \mathrm{mM}$ succinate, $\mathrm{pH}$ 9.0. SC was similarly

264 transfected into CHO DP12 cells and purified as described (Kumar et al., 2020; Wong et

265 al., 2010). Briefly, media containing secreted SC was applied to Ni Sepharose Excel

266 resin (GE Healthcare, Chicago, IL), washed with buffer B (50 mM NaPO4, pH 7.4, 200

$267 \mathrm{mM} \mathrm{naCl}$, and $1 \mathrm{mM} \mathrm{NaN}_{3}$ ), and eluted with buffer B supplemented with $400 \mathrm{mM}$

268 Imidazole. Fractions containing IgM-Fc/JC complexes or SC were concentrated with a

269 100- or 30-kDa MWCO Amicon centrifugal filtration device, respectively, prior to loading

270 to a HiLoad Superdex 200 pg 26/60 column (GE Healthcare) equilibrated with buffer C

271 (20 mM HEPES, pH 7.2, $250 \mathrm{mM} \mathrm{NaCl}$ ). Peak fractions corresponding to pentameric

272 IgM-Fc/JC complex or SC were recovered and concentrated.

273

274 CryoEM Sample Preparation and Data Collection

275 Purified IgM-Fc/JC assemblies at 1 uM were complexed with 2-fold molar excess of SC

276 in the presence of $0.3 \mathrm{mM}$ reduced Glutathione (Sigma, St. Louis, MO) and incubated

277 overnight at $4^{\circ} \mathrm{C}$. IgM-Fc/JC/SC complexes were separated from excess SC via size 
278 exclusion chromatography using a Superose 6 increase 3.2/300 (GE Healthcare) in

279 Buffer $\mathrm{C}$ described above. Peak fractions corresponding to complexes were collected

280 and diluted to $0.2 \mathrm{mg} / \mathrm{mL}$. Prior to cryo-grid preparation, samples were briefly cross-

281 linked with $0.05 \%$ glutaraldehyde (Sigma) for 10 mins at $20{ }^{\circ} \mathrm{C}$. Cryo-grids were

282 prepared as previously described (Kumar et al., 2020). Briefly, Quantifoil R 0.6/1 holey

283 carbon grids were freshly glow discharged for 5 s prior with a Solarus 950 Plasma

284 Cleaner (Gatan, Pleasanton, CA) prior to application of 3 uL protein sample. Grids were

285 blotted with Vitrobot Filter Paper (Electron Microscopy Sciences, Hatfield, PA) for $2.5 \mathrm{~s}$

286 with a blot force of 8 using a Vitrobot Mark IV (Thermo Fisher, Waltham, MA) set to $4^{\circ} \mathrm{C}$

287 with 100\% humidity, and plunged into liquid nitrogen-cooled liquid ethane. Movie stacks

288 were collected on a Titan Krios (Thermo Fisher) operating at $300 \mathrm{kV}$ equipped with K2

289 Summit (Gatan) direct electron detector using SerialEM (Mastronarde, 2005). Images

290 were collected at $165,000 X$ calibrated to a pixel size of $0.824 \AA$ Apixel. Movie stacks

291 containing 50 images were recorded every $0.2 \mathrm{~s}$ with a total electron exposure of 53.02

292 electrons/Å2.

293

294 Data Processing

29518,168 movie stacks at zero-degree tilt were collected, along with 5,866 movie stacks

296 collected with 40-degree tilt. Movie stacks were imported and processed using the

297 cisTEM processing pipeline (Grant et al., 2018). Briefly, motion-corrected and aligned

298 micrographs with CTF fits better than $4.5 \AA$ were filtered prior to auto-picking with an

299 inter-particle exclusion radius of $90 \AA$ and particle radius of $60 \AA$, with a threshold height 
300 of one standard deviation above noise in cisTEM. One round of 2D classification was

301 performed with 150 classes for particles from zero-degree tilt data and 100 classes for

302 particles from 40-degree tilt datasets. Classes were manually inspected and selected for

303 ab initio model generation, followed by 3D auto-refinement. To improve the quality of

304 the reconstruction, a mask excluding the peripheral $\lg \mathrm{M} \mathrm{C} \mu 2$ was applied, followed by

305 3D-classification with 5 classes. One class containing the best map features was

306 selected for iterative 3D refinement. Additional focused refinements around IgM-Fc C $\mu 4$

307 and SC-D1, D3-5 or IgM-Fc1 and SC-D1 domains were performed to improve map

308 quality in those regions. Focus refined maps were combined using

309 phenix.Combine_focused_maps (Liebschner et al., 2019). Local resolution was

310 determined using ResMap (Kucukelbir et al., 2013) and rendered on a linear scale from

311 2.6-5.0 Å in UCSF Chimera (Pettersen et al., 2004).

313 Model Building, Refinement, and Validation

314 Homology models of human $\operatorname{lgM} \mathrm{C} \mu 3$ and $\mathrm{C} \mu 4$ domains were built based on the NMR

315 structure of murine $\mathrm{C} \mu 3$ (PDB 4BA8) and crystal structure of murine C $\mu 4$ (PDB 4JVW)

316 using SWISS-MODEL (Waterhouse et al., 2018). Homology models of IgM tailpiece

317 segments were similarly generated from the cryoEM structure of pentameric slgA (PDB

318 6UEA). Homology models, along with the JC and individual domains of the SC from

319 pentameric slgA, were docked and rigid-body fit into the map using

320 phenix.realspacerefine (Afonine et al., 2018). The model was subsequently inspected

321 and further improved through iterative model building in Coot (Emsley et al., 2010) and 
322 real-space refinement in Phenix. Models were validated using phenix.validation_cryoem

323 (Afonine et al., 2018) as well as EMRinger (Barad et al., 2015). Figures were generated

324 using PyMOL (The PyMOL Molecular Graphics System, Version 2.2.2 Schrödinger,

325 LLC) and UCSF Chimera (Pettersen et al., 2004) and ChimeraX (Goddard et al., 2017).

327 Sequence Alignments and Conservation Analysis

328 Sequences for human IgM-Fc (342-576), IgA1-Fc (241-472), and IgA2-Fc (241-472)

329 were aligned using Clustal Omega with default settings (Madeira et al., 2019), and the

330 results rendered by conservation index on structure of IgM-FC/JC in PyMOL.

Acknowledgements: We thank Christine Tam, Yvonne Franke, and the Biomolecular

334 Resource group for construct generation and the Research Materials Group for protein

335 expression. We thank members of the Structural Biology department and the cryo-EM

336 group for helpful discussions, including Alexis Rohou for advice on data processing and

337 Jian Payandeh for helpful discussions on manuscript preparation.

339 Funding: This work was funded and conducted by Genentech, Inc.

341 Competing Interests: All authors are employees of Genentech, Inc., a member of the

342 Roche Group, and may hold stock and options. 
344 Data and materials availability: The cryo-EM map and coordinate model have been

345 deposited to the Worldwide Protein Data Bank with PDB accession code 7KOC and

346 EMDB code EMD-22591.

\section{References}

Afonine PV, Klaholz BP, Moriarty NW, Poon BK, Sobolev OV, Terwilliger TC, Adams PD, Urzhumtsev A. 2018. New tools for the analysis and validation of cryo-EM maps and atomic models. Acta Crystallogr Sect D 74:814-840. doi:10.1107/s2059798318009324

Barad BA, Echols N, Wang RY-R, Cheng Y, DiMaio F, Adams PD, Fraser JS. 2015. EMRinger: side chain-directed model and map validation for 3D cryo-electron microscopy. Nat Methods 12:943-946. doi:10.1038/nmeth.3541

Catanzaro JR, Strauss JD, Bielecka A, Porto AF, Lobo FM, Urban A, Schofield WB, Palm NW. 2019. IgA-deficient humans exhibit gut microbiota dysbiosis despite secretion of compensatory IgM. Sci Rep-uk 9:13574. doi:10.1038/s41598-01949923-2

Coyne RS, Siebrecht M, Peitsch MC, Casanova JE. 1994. Mutational analysis of polymeric immunoglobulin receptor/ligand interactions. Evidence for the involvement of multiple complementarity determining region (CDR)-like loops in receptor domain I. J Biological Chem 269:31620-5.

Davis AC, Roux KH, Pursey J, Shulman MJ. 1989. Intermolecular disulfide bonding in IgM: effects of replacing cysteine residues in the mu heavy chain. Embo J 8:25192526. doi:10.1002/j.1460-2075.1989.tb08389.x

372 Goddard TD, Huang CC, Meng EC, Pettersen EF, Couch GS, Morris JH, Ferrin TE. 373 2017. UCSF ChimeraX: Meeting modern challenges in visualization and analysis. Protein Sci Publ Protein Soc 27:14-25. doi:10.1002/pro.3235 
375

Grant T, Rohou A, Grigorieff N. 2018. cisTEM, user-friendly software for single-particle image processing. Elife 7:e35383. doi:10.7554/elife.35383

Hamburger AE, West AP, Bjorkman PJ. 2004. Crystal Structure of a Polymeric Immunoglobulin Binding Fragment of the Human Polymeric Immunoglobulin Receptor. Structure 12:1925-1935. doi:10.1016/j.str.2004.09.006

Heyman B, Shulman MJ. 2016. Structure, Function, and Production of Immunoglobulin M (IgM). Struct Funct Divers Recept 1-14. doi:10.1016/b978-0-12-374279-7.05001-3

Hiramoto E, Tsutsumi A, Suzuki R, Matsuoka S, Arai S, Kikkawa M, Miyazaki T. 2018. The IgM pentamer is an asymmetric pentagon with an open groove that binds the AIM protein. Sci Adv 4:eaau1199. doi:10.1126/sciadv.aau1199

Johansen, Braathen, Brandtzaeg. 2000. Role of J Chain in Secretory Immunoglobulin Formation. Scand J Immunol 52:240 248. doi:10.1046/j.1365-3083.2000.00790.x

Johansen F-E, Schjerven H, Norderhaug IN, Brandtzaeg P. 1999. Regulation of the Formation and External Transport of Secretory Immunoglobulins. Critical Rev Immunol 19:28. doi:10.1615/critrevimmunol.v19.i5-6.50

Kaetzel CS. 2005. The polymeric immunoglobulin receptor: bridging innate and adaptive immune responses at mucosal surfaces. Immunol Rev 206:83-99. doi:10.1111/j.0105-2896.2005.00278.x

Klimovich VB. 2011. IgM and its receptors: Structural and functional aspects. Biochem Mosc 76:534 549. doi:10.1134/s0006297911050038

Kownatzki E, Drescher M. 1973. Antigen binding and complement fixing activity of IgM molecules reassociated in the presence and absence of $\mathrm{J}$ chains. Clin Exp Immunol 15:557-63.

Kucukelbir A, Sigworth FJ, Tagare HD. 2013. Quantifying the local resolution of cryoEM density maps. Nat Methods 11:63-65. doi:10.1038/nmeth.2727

Kumar N, Arthur CP, Ciferri C, Matsumoto ML. 2020. Structure of the secretory immunoglobulin A core. Science 367:1008-1014. doi:10.1126/science.aaz5807

Li Y, Wang G, Li N, Wang Y, Zhu Q, Chu H, Wu W, Tan Y, Yu F, Su X-D, Gao N, Xiao J. 2020. Structural insights into immunoglobulin M. Science eaaz5425. doi:10.1126/science.aaz5425

Liebschner D, Afonine PV, Baker ML, Bunkóczi G, Chen VB, Croll TI, Hintze B, Hung LW, Jain S, McCoy AJ, Moriarty NW, Oeffner RD, Poon BK, Prisant MG, Read RJ, Richardson JS, Richardson DC, Sammito MD, Sobolev OV, Stockwell DH, Terwilliger 
TC, Urzhumtsev AG, Videau LL, Williams CJ, Adams PD. 2019. Macromolecular structure determination using X-rays, neutrons and electrons: recent developments in Phenix. Acta Crystallogr Sect D 75:861-877. doi:10.1107/s2059798319011471

Liu J, Wang Y, Xiong E, Hong R, Lu Q, Ohno H, Wang J-Y. 2019. Role of the IgM Fc Receptor in Immunity and Tolerance. Front Immunol 10:529. doi:10.3389/fimmu.2019.00529

Lombana TN, Rajan S, Zorn JA, Mandikian D, Chen EC, Estevez A, Yip V, Bravo DD, Phung W, Farahi F, Viajar S, Lee S, Gill A, Sandoval W, Wang J, Ciferri C, Boswell CA, Matsumoto ML, Spiess C. 2019. Production, characterization, and in vivo half-life extension of polymeric IgA molecules in mice. Mabs.

doi:10.1080/19420862.2019.1622940

Longet S, Miled S, Lötscher M, Miescher SM, Zuercher AW, Corthésy B. 2012. Human Plasma-derived Polymeric IgA and IgM Antibodies Associate with Secretory Component to Yield Biologically Active Secretory-like Antibodies. J Biol Chem 288:4085-4094. doi:10.1074/jbc.m112.410811

Madeira F, Park Y mi, Lee J, Buso N, Gur T, Madhusoodanan N, Basutkar P, Tivey ARN, Potter SC, Finn RD, Lopez R. 2019. The EMBL-EBI search and sequence analysis tools APIs in 2019. Nucleic Acids Res 47:W636-W641. doi:10.1093/nar/gkz268

Mastronarde DN. 2005. Automated electron microscope tomography using robust prediction of specimen movements. J Struct Biol 152:36-51. doi:10.1016/j.jsb.2005.07.007

Pettersen EF, Goddard TD, Huang CC, Couch GS, Greenblatt DM, Meng EC, Ferrin TE. 2004. UCSF Chimera?A visualization system for exploratory research and analysis. J Comput Chem 25:1605-1612. doi:10.1002/jcc.20084

Sharp TH, Boyle AL, Diebolder CA, Kros A, Koster AJ, Gros P. 2019. Insights into IgMmediated complement activation based on in situ structures of IgM-C1-C4b. Proc National Acad Sci 201901841. doi:10.1073/pnas.1901841116

Sørensen V, Rasmussen IB, Norderhaug L, Natvig I, Michaelsen TE, Sandlie I. 1996. Effect of the $\operatorname{lgM}$ and $\lg A$ secretory tailpieces on polymerization and secretion of $\lg M$ and IgG. J Immunol Baltim Md 1950 156:2858-65.

Sørensen V, Sundvold V, Michaelsen TE, Sandlie I. 1999. Polymerization of IgA and IgM: Roles of Cys309/Cys414 and the Secretory Tailpiece. Journal of immunology (Baltimore, Md : 1950) 162:3448 3455. 
442 Stadtmueller BM, Huey-Tubman KE, López CJ, Yang Z, Hubbell WL, Bjorkman PJ.

443 2016. The structure and dynamics of secretory component and its interactions with

444 polymeric immunoglobulins. Elife 5:213. doi:10.7554/elife.10640

445 Wang Y, Wang G, Li Y, Zhu Q, Shen H, Gao N, Xiao J. 2020. Structural insights into 446 secretory immunoglobulin $A$ and its interaction with a pneumococcal adhesin. Cell 447 Res 30:602-609. doi:10.1038/s41422-020-0336-3

448 Waterhouse A, Bertoni M, Bienert S, Studer G, Tauriello G, Gumienny R, Heer FT, 449 de Beer TAP, Rempfer C, Bordoli L, Lepore R, Schwede T. 2018. SWISS-MODEL: 450 homology modelling of protein structures and complexes. Nucleic Acids Res

451 46:W296-W303. doi:10.1093/nar/gky427

452 Wong AW, Baginski TK, Reilly DE. 2010. Enhancement of DNA uptake in FUT8-deleted $453 \mathrm{CHO}$ cells for transient production of afucosylated antibodies. Biotechnol Bioeng

$454 \quad$ 106:751-763. doi:10.1002/bit.22749 
bioRxiv preprint doi: https://doi.org/10.1101/2020.09.10.291138; this version posted September 11, 2020. The copyright holder for this preprint (which was not certified by peer review) is the author/funder, who has granted bioRxiv a license to display the preprint in perpetuity. It is made available under aCC-BY 4.0 International license.

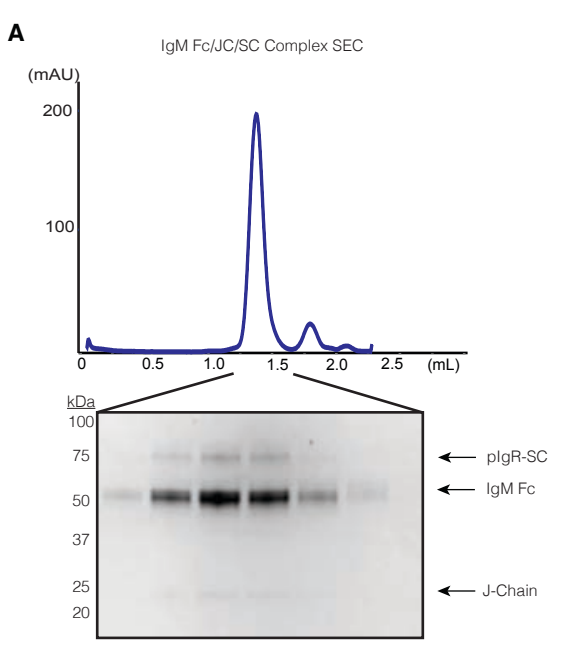

F
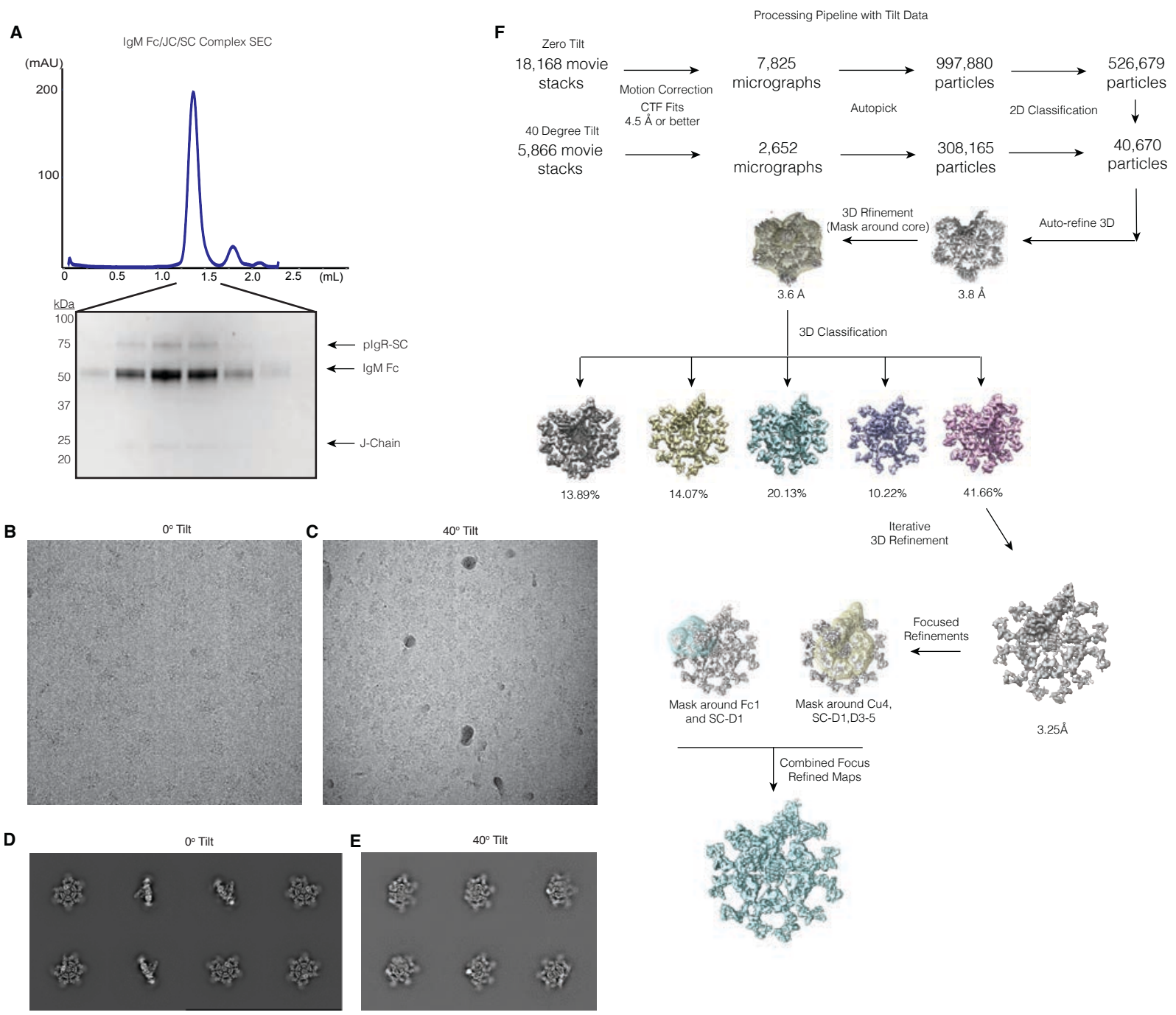

G

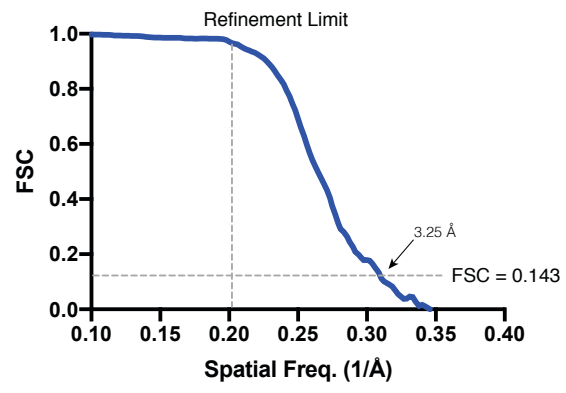

H

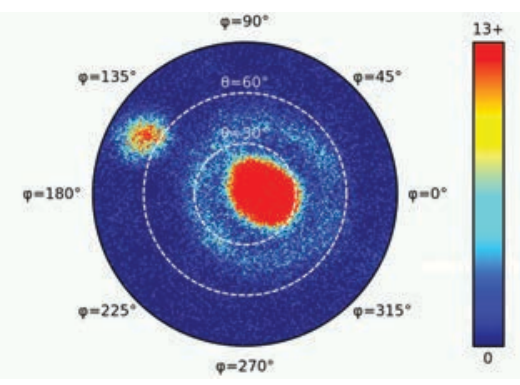

Figure 1 - figure supplement 1: SIgM purification and cryo-EM data processing. (A) Representative size-exclusion chromatography trace (top) and reducing SDS-PAGE gel (bottom) of pentameric lgM-Fc/JC/SC complex. Representative motion-corrected and aligned cryo-EM images of samples imaged with zero degree (B) and 40 degree tilt $(C)$ along with selected corresponding 2D class averages for zero degree (D) and 40 degree tilt $(E)$ datasets. Summarized data processing pipeline along $(F)$, along with FSC curve for the final reconstruction $(G)$, with refinement limit indicated by the dashed vertical line and $F S C=0.143$ indicated by the dashed horizontal line. $(\mathrm{H})$ Corresponding angular distribution plot for reconstruction shown in $(\mathrm{G})$. 
bioRxiv preprint doi: https://doi.org/10.1101/2020.09.10.291138; this version posted September 11, 2020. The copyright holder for this preprint (which was not certified by peer review) is the author/funder, who has granted bioRxiv a license to display the preprint in perpetuity. It is made available under aCC-BY 4.0 International license.

A

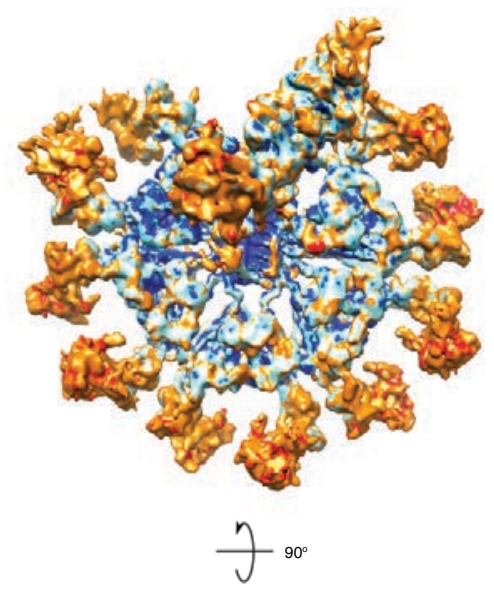

B
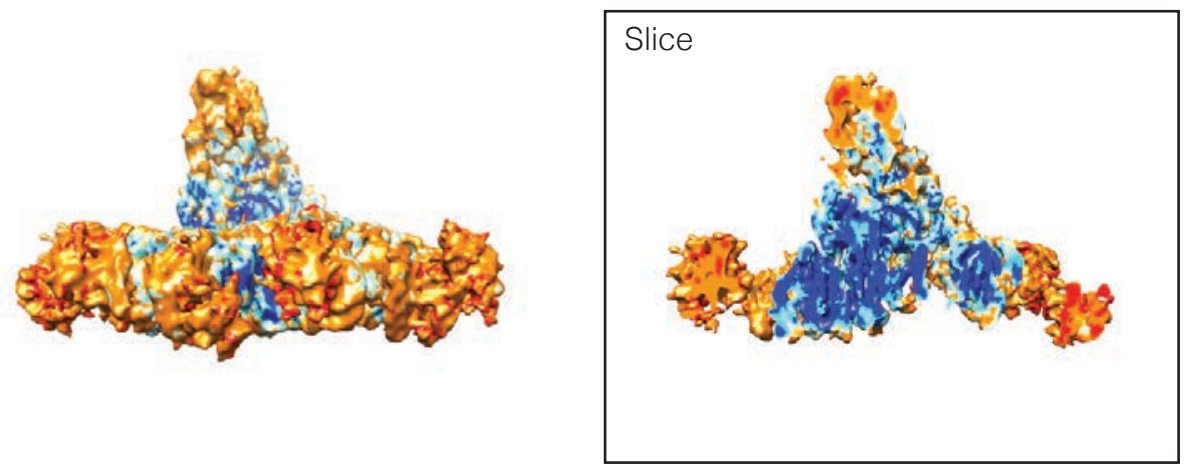

Resolution $(\AA)$
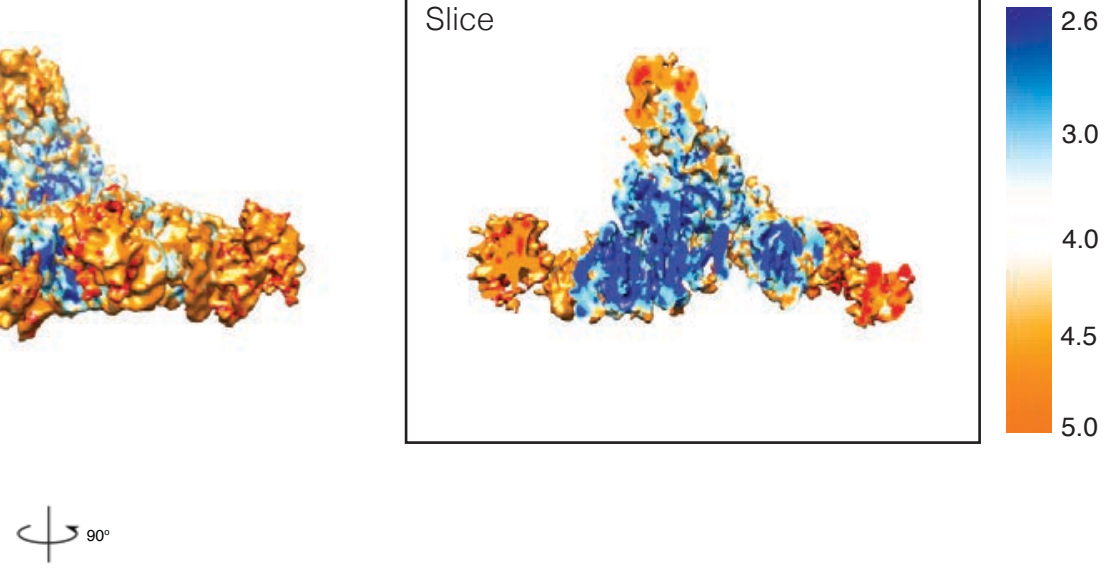

C

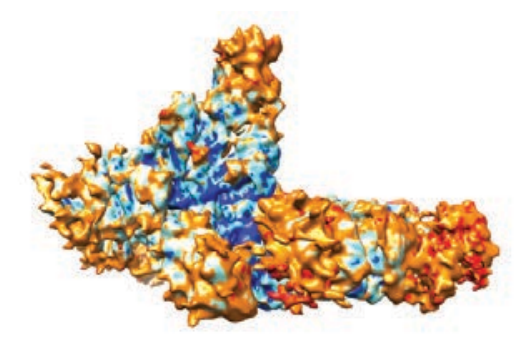

Figure 1 - figure supplement 2: Local resolution of the slgM reconstruction. Top (A), front (B), and side (C) views of the IgM reconstruction colored by local resolution from blue to red. Slice through SC-D1 and Fc interface from front view in (B) shown on right. 
bioRxiv preprint doi: https://doi.org/10.1101/2020.09.10.291138; this version posted September 11, 2020. The copyright holder for this preprint (which was not certified by peer review) is the author/funder, who has granted bioRxiv a license to display the preprint in perpetuity. It is made available under aCC-BY 4.0 International license.

A

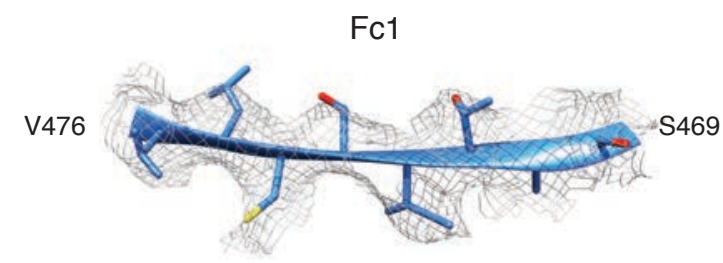

Fc1
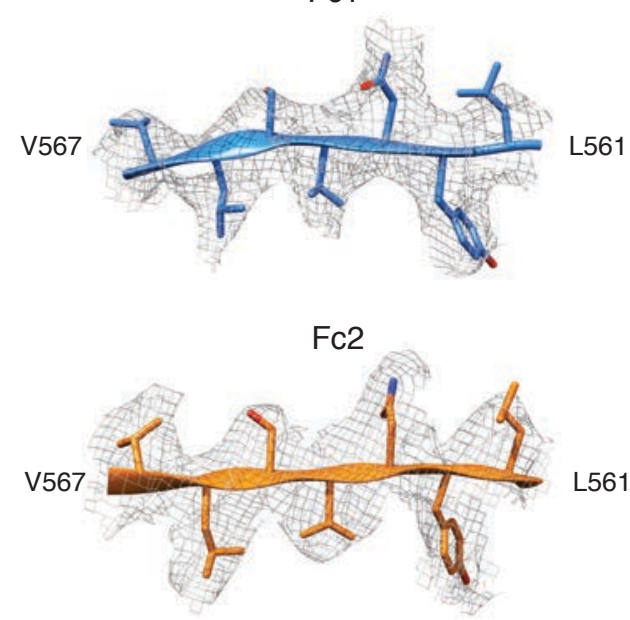

B

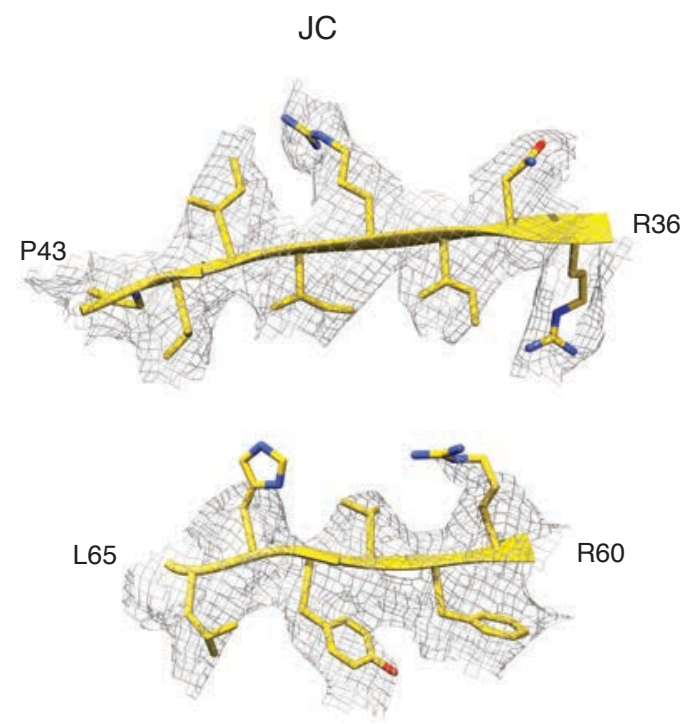

C

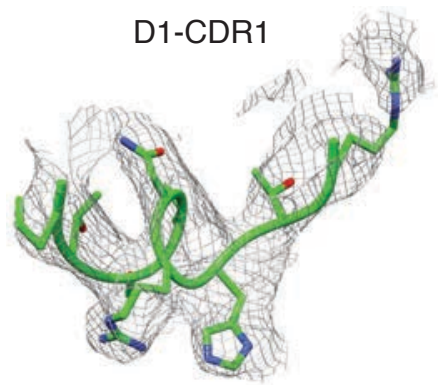

D1-CDR2

D1-CDR3
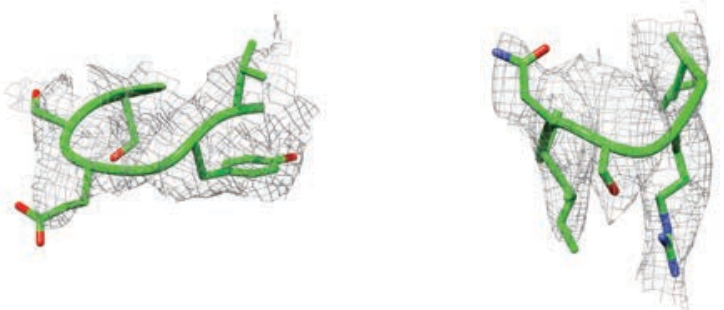

Figure 1 - figure supplement 3: Cryo-EM maps of select regions of slgM complex. (A) Density of $\beta$-strands corresponding to IgM Fc1 and Fc2. (B) Density corresponding to select regions of the JC. (C) Density corresponding to SC-D1 CDR1, 2, and 3. 
bioRxiv preprint doi: https://doi.org/10.1101/2020.09.10.291138; this version posted September 11, 2020. The copyright holder for this preprint (which was not certified by peer review) is the author/funder, who has granted bioRxiv a license to display the preprint in perpetuity. It is made available under aCC-BY 4.0 International license.

A SP $|P 01871|$ IGHM_HUMAN
SP |P01876| IGHA1_HUMAN
SP $\mid$ P01877| IGHA2_HUMAN

SP|P01871|IGHM_HUMAN SP $\mid$ P01876| IGHA 1 HUMAN SP |P01877| IGHA2_HUMAN

SP |P01871|IGHM_HUMAN SP $\mid$ P01876| IGHA 1 _HUMAN SP $\mid$ P01877|IGHA2_HUMAN

SP $\mid$ P01871 | IGHM HUMAN SP $\mid$ P01876| IGHA $\overline{1}$ HUMAN SP |P01877| IGHA2_HUMAN

B

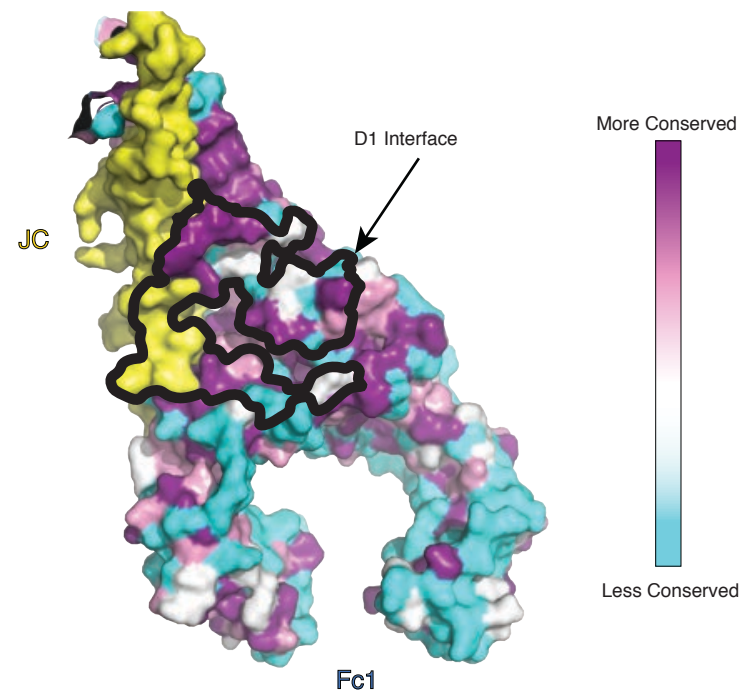

$\mathrm{C} \mu 3 / \mathrm{Ca} 2$

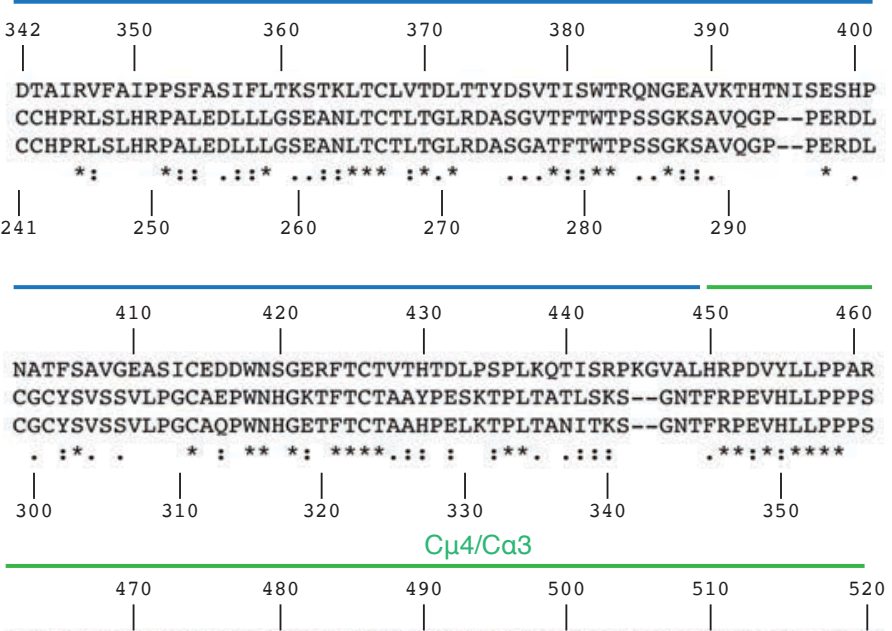

EQLNLRESATITCLVTGFSPADVFVQWMQRGQPLSPEKYVTSAPMPEPQAPG-RYFAHSI EELALNELVTLTCLARGF SPKDVLVRWLQGSQELPREKYLTWASRQEPSQGTTTFAVTSI EELALNELVTLTCLARGFSPKDVLVRWLQGSQELPREKYLTWASRQEPSQGTTTYAVTSI

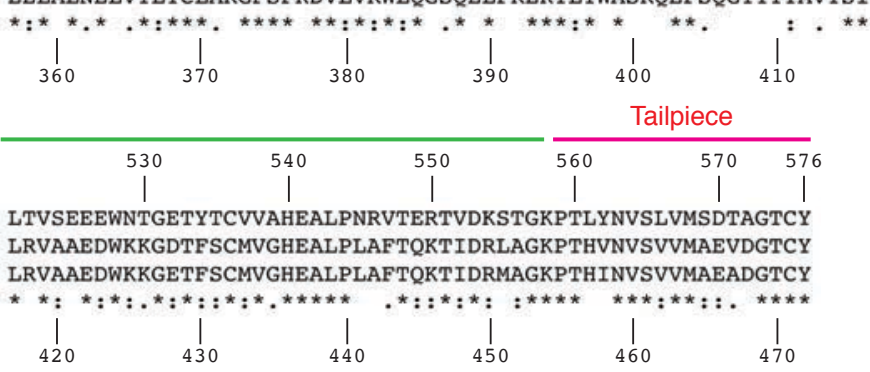

Figure 2 - figure supplement 1: Sequence alignments and surface conservation of SC-D1 interface with IgM-Fc/JC. (A) Clustal Omega multiple sequence alignments of human IgM residues 342-576 (UniProt P01871), human IgA1 residues 241-472 (UniProt P01876), and human IgA2 residues 241-472 (UniProt P01877). (B) Top-down view of IgM-Fc/JC colored by sequence conservation with IgA-Fc with SC-D1 omitted for clarity. Interaction interface of SC-D1 is outlined on the surface in black. Conservation index: Identical residues (maroon); residues with highly similar chemical properties (pink); residues with weakly similar chemical properties (white); dissimilar residues (cyan). 
462 Table 1: CryoEM data collection, refinement, and validation statistics.

\begin{tabular}{|c|c|}
\hline & $\begin{array}{l}\text { Pentameric slgM } \\
\text { (PDB ID 7KOC) }\end{array}$ \\
\hline \multicolumn{2}{|l|}{ Data collection } \\
\hline Microscope & Titan Krios \\
\hline Voltage (kV) & 300 \\
\hline Detector & K2 Summit \\
\hline Data collection software & SerialEM \\
\hline Energy filter & Bioquantum (20 eV slit) \\
\hline Magnification & $165,000 \mathrm{X}$ \\
\hline Exposure rate $\left(\mathrm{e}^{\left.-/ \AA^{2} / \mathrm{s}\right)}\right.$ & 5.30 \\
\hline Frame rate $(\mathrm{s})$ & 0.2 \\
\hline Total frames per movie & 50 \\
\hline Pixel size $(\AA)$ & 0.824 \\
\hline Total electron exposure $\left(\mathrm{e}-/ \AA^{2}\right)$ & 53.02 \\
\hline Defocus range $(\mu \mathrm{m})$ & -0.6 to -1.8 \\
\hline \multicolumn{2}{|l|}{ Data processing } \\
\hline Processing software & cisTEM \\
\hline Number of micrographs & 24,034 \\
\hline Number of extracted particles & $1,306,045$ \\
\hline Symmetry & C1 \\
\hline Number of refined particles & 244,123 \\
\hline $\begin{array}{l}\text { Map resolution }(\AA) \text { at: } \\
\text { FSC } 0.5 \text { (unmasked/masked) } \\
\text { FSC } 0.143 \text { (unmasked/masked) }\end{array}$ & $\begin{array}{l}4.5 / 4.1 \\
3.4 / 3.2\end{array}$ \\
\hline Map resolution range $(\AA)$ & $2.6-6.0$ \\
\hline \multicolumn{2}{|l|}{ Refinement } \\
\hline Software & PHENIX v1.18.2 \\
\hline Models used (PDB code) & 4BA8, 4JVW, 6UEA \\
\hline Map sharpening B factor $\left(\AA^{2}\right)$ & -150 \\
\hline Map correlation coefficient & 0.74 \\
\hline \multicolumn{2}{|l|}{ Model composition } \\
\hline Non-hydrogen atoms & 22,540 \\
\hline Protein residues & 2,898 \\
\hline N-linked glycans & 2 \\
\hline \multicolumn{2}{|l|}{ R.M.S deviations } \\
\hline Bond lengths $(\AA ̊)$ & 0.988 \\
\hline Bond angles ( $\left.{ }^{\circ}\right)$ & 0.007 \\
\hline \multicolumn{2}{|l|}{ Ramachandran Plot } \\
\hline Preferred (\%) & 85.0 \\
\hline Allowed (\%) & 15.0 \\
\hline Outliers (\%) & 0.0 \\
\hline \multicolumn{2}{|l|}{ Validation } \\
\hline MolProbity score & 2.66 \\
\hline Clash score & 16.78 \\
\hline Poor rotamers $(\%)$ & 0.08 \\
\hline $\mathrm{C} \beta$ outliers (\%) & 0 \\
\hline CaBLAM outliers (\%) & 6.24 \\
\hline
\end{tabular}

